\title{
Comments on the compatibility of thermodynamic equilibrium conditions with lattice propagators
}

\author{
Fabrizio Canfora ${ }^{1, \mathrm{a}}$, Alex Giacomini ${ }^{2, \mathrm{~b}}$, Pablo Pais ${ }^{1,3, \mathrm{c}}$, Luigi Rosa $^{4,5, \mathrm{~d}}$, Alfonso Zerwekh ${ }^{6, \mathrm{e}}$ \\ ${ }^{1}$ Centro de Estudios Científicos (CECs), Casilla 1469, Valdivia, Chile \\ ${ }^{2}$ Instituto de Ciencias Físicas y Matemáticas, Universidad Austral de Chile, Casilla 567, Valdivia, Chile \\ ${ }^{3}$ Physique Théorique et Mathématique, Université Libre de Bruxelles and International Solvay Institutes, Campus Plaine C.P. 231, 1050 Brussels, \\ Belgium \\ ${ }^{4}$ Dipartimento di Fisica, Universitá di Napoli Federico II, Complesso Universitario di Monte S. Angelo, Via Cintia Edificio 6, 80126 Naples, Italy \\ 5 INFN, Sezione di Napoli, Complesso Universitario di Monte S. Angelo, Via Cintia Edificio 6, 80126 Naples, Italy \\ ${ }^{6}$ Departamento de Física and Centro Científico-Tecnológico de Valparaíso, Universidad Técnica Federico Santa María, \\ Casilla 110-V, Valparaiso, Chile
}

Received: 20 June 2016 / Accepted: 23 July 2016 / Published online: 8 August 2016

(C) The Author(s) 2016. This article is published with open access at Springerlink.com

\begin{abstract}
In this paper the compatibility is analyzed of the non-perturbative equations of state of quarks and gluons arising from the lattice with some natural requirements for selfgravitating objects at equilibrium: the existence of an equation of state (namely, the possibility to define the pressure as a function of the energy density), the absence of superluminal propagation and Le Chatelier's principle. It is discussed under which conditions it is possible to extract an equation of state (in the above sense) from the non-perturbative propagators arising from the fits of the latest lattice data. In the quark case, there is a small but non-vanishing range of temperatures in which it is not possible to define a single-valued functional relation between density and pressure. Interestingly enough, a small change of the parameters appearing in the fit of the lattice quark propagator (of around $10 \%$ ) could guarantee the fulfillment of all the three conditions (keeping alive, at the same time, the violation of positivity of the spectral representation, which is the expected signal of confinement). As far as gluons are concerned, the analysis shows very similar results. Whether or not the non-perturbative quark and gluon propagators satisfy these conditions can have a strong impact on the estimate of the maximal mass of quark stars.
\end{abstract}

\footnotetext{
a e-mail: fcanforat@gmail.com

b e-mail: alexgiacomini@uach.cl

ce-mail: pais@cecs.cl

de-mail: rosa@na.infn.it

e e-mail: alfonso.zerwekh@usm.cl
}

\section{Introduction}

One of the main open problems in theoretical physics is a proper understanding of the infrared behavior of non-Abelian gauge theories, like Quantum Chromodynamics (QCD), and of its phase diagram (see [1]). The non-perturbative nature of the infrared region of QCD prevents one from using the standard perturbative techniques based on Feynman diagrams. Thus, it is necessary to rest on non-perturbative techniques and/or lattice data. In the present paper, we will combine lattice data (both for the quarks and gluons propagators) together with the non-perturbative effects arising from (the elimination of) Gribov copies [2] (for the gluonic sector) to extract non-perturbative equations of state for quarks as well as gluons. We will adopt the $\zeta$-function regularization technique [3-5], which allows one to write many of the physical quantities in a closed form as (very rapidly convergent) series of Bessel functions. This technical point will play an important role in the following.

The details of non-perturbative propagators of quarks and gluons are of great interest in applications. For instance, in astrophysics, there is evidence supporting the existence of quark stars (two detailed reviews are [6,7]). As such objects are gravitating, it is of fundamental importance to check under which conditions they can be in hydrostatic equilibrium and which is the expected upper bound on their mass. Due to the great difficulty to construct analytically an equation of state (EOS) for such extreme matter configurations, it is important to have some estimates on the mass bounds of self-gravitating objects which can be deduced by generic principles rather than from the exact form of the EOS. For a neutron star this has been done by Rhoades and Ruffini [8] 
using only very basic principles. Two of the required basic principles are the absence of superluminal propagation and Le Chatelier's principle.

The absence of superluminal propagation is necessary to enforce causality and is considered one of the most basic principles of relativistic physics.

Le Chatelier's principle simply states that when any system at equilibrium is subjected to change, it will react in such a way to oppose to the change. This means that assuming Le Chatelier's principle is completely generic as, without it, it would not even be possible have a stable equilibrium.

The third principle required by Rhoades and Ruffini is the validity of the hydrostatic equilibrium equation of General Relativity. Just from the above requirements, they were able to derive a bound of $3.2 M_{\odot}$ for a neutron star without knowing any further details of the EOS (besides its existence of course, as will be explained in a moment).

As it is possible to extract detailed information as regards the non-perturbative propagators from lattice data (as well as from the Gribov-Zwanziger procedure) a natural question arises: will the equations of state derived from the nonperturbative quarks and gluons propagators be compatible with the basic principles stated above?

In the present analysis, in order to be as generic as possible, we will drop the assumption of the hydrostatic equilibrium equation as it is specific to General Relativity (it may be that this theory acquires some corrections in some extreme range of parameters). The other two principles, namely causality and Le Chatelier's principle are, instead, quite generic and, consequently, we will consider them.

Actually, before discussing these two principles, there is a more basic requirement which was implicitly assumed in [8] (as well as in a great part of the theoretical literature on gravitating compact objects): namely the existence of a well-defined EOS or, in other words, the possibility to define an implicit one-to-one functional relation between pressure and energy density. The importance of such a requirement becomes obvious if one considers that, if it is not satisfied, it is not even possible to discuss the coupling with the Einstein equations (in the usual way, at least). That is the reason why in [8] such a principle was not analyzed in detail, rather it was just considered as obvious. However, the present analysis shows that the very existence of an EOS depends on the precise values of the parameters appearing in the fit of the lattice propagators. Remarkably, there are cases in which it is not possible at all to extract a well-defined EOS from the non-perturbative propagators. This, for instance, prevents one from coupling strongly interacting quarks and gluons to Einstein gravity in any obvious way. In particular, in these situations the bound derived in [8] would not apply. Usually, the fact that it is not possible to define a one-to-one relation between pressure and energy density suggests that some extra physical parameter is needed to properly label the equilibrium states in order to define an EOS in the usual sense.

The fact that there may not exist a well-defined EOS in certain situations can be seen as follows. From the nonperturbative propagator it is possible to compute the grand partition function from which all relevant thermodynamical quantities like the pressure and energy density as functions of the temperature and of the chemical potential can be derived. Taking, for example, a fixed value of the chemical potential it is possible to get a parametric equation for the energy density $e(T)$ versus pressure $P(T)$ curve. This curve, however, represents a single-valued function $P=P(e)$ only when the functions $P(T)$ and $e(T)$ are strictly monotonous as a function of the temperature $T$ or the shapes and ranges of non monotonicity in $T$ for $P$ and $e$ are exactly the same.

The computation of the grand partition function from the non-perturbative lattice quarks propagator at finite temperature and chemical potential has been performed in the reference [9] using dimensional regularization techniques. In this paper, the $\zeta$-function regularization technique is used instead and the non-perturbative gluons propagator is discussed as well. The advantage of the $\zeta$-function regularization $[10,11]$ is that it reduces the number of numerical integrations (many of the expressions are evaluated as fast converging series of Bessel functions) and, consequently, it reduces the numerical error allowing to see even very tiny effects. Indeed, although our computations show that the two techniques clearly agree, using the $\zeta$-function approach it is possible to see that for the values of the parameters appearing in the fit of the last lattice quarks propagator, the curve $P(T)$ and $e(T)$ of the pressure and energy density as a function of the temperature are non-strictly monotonic functions and have different shapes near $T=0$. This means that for a small range of temperatures, the functional relation of pressure and energy density is not one-to-one: namely, one of the requirements of Rhoades and Ruffini is violated.

The problem in the EOS being well defined disappears by allowing a change in the fit parameters of around $10 \%$. Interestingly, once the existence of an EOS has been ensured, the causality and Le Chatelier principles turn out to be almost satisfied without any extra requirements on the parameters. For this reason, it may be interesting to explore the phenomenological consequences of allowing such changes in the fit parameters.

A similar analysis reveals that the same requirement of Rhoades and Ruffini can be violated in the case of the nonperturbative gluons EOS. The theoretical interest of the gluon case is that if one considers a Gribov-Zwanziger (GZ) propagator with the inclusion of the effects of the condensates then, by allowing a change in the fit parameters of around $10 \%$, one can satisfy all the requirements of Rhoades and Ruffini. On the other hand, if one does not include the condensates, 
then a change in the Gribov mass of around $10 \%$ is definitely not enough to satisfy all the requirements of Rhoades and Ruffini.

The paper is organized as follows: in the next section the relevant thermodynamic quantities for the non-perturbative quark propagator are constructed. The most relevant details of the computations related to the $\zeta$-function regularization technique are kept in the main text as they are fundamental in detecting the non-existence regime of the EOS. In the third section, the analysis is extended to the gluonic sector. The last section is dedicated to the conclusions and perspectives.

\section{The non-perturbative quark propagator and its thermodynamics}

Let us start the analysis with the non-perturbative quark propagator $S(p)$ arising from the lattice $[12,13]$

$S(p)=-\frac{\gamma_{\mu} p_{\mu}+\mathbf{1}_{4} M_{0}(p)}{p^{2}+M_{0}^{2}(p)}, \quad M_{0}(p)=\frac{M_{3}}{p^{2}+m^{2}}+m_{0}$,

$M_{3}=0.196 \mathrm{GeV}^{3}, m^{2}=0.639 \mathrm{GeV}^{2}, m_{0}=0.014 \mathrm{GeV}$,

where $\gamma_{\mu}$ are the Euclidean Dirac matrices and $\mathbf{1}_{4}$ is the $4 \times 4$ identity matrix. Such a propagator can be included in the framework of the so-called refined Gribov-Zwanziger (RGZ) approach as indicated in [14,15]. It is worth emphasizing here that the above propagator can be expanded into three 'standard' Fermions propagators, two of which having complex conjugated poles and the third with real poles. The physical interpretation of having complex conjugated poles is that they are a signal of confinement as propagators with complex poles in $p^{2}$ violate positivity. Such poles are determined by the following equation:

$$
\begin{gathered}
p^{2}\left(p^{2}+m^{2}\right)^{2}+\left[M_{3}+m_{0}\left(p^{2}+m^{2}\right)\right]^{2} \\
=\left(p^{2}+\alpha_{1}\right)\left(p^{2}+\alpha_{2}\right)\left(p^{2}+\alpha_{3}\right),
\end{gathered}
$$

meaning that the set $\left\{\alpha_{i}\right\}$ represents minus the roots of the denominator in $S(p)$.

\subsection{Thermodynamics}

As said above, the idea to compute the grand partition function using the non-perturbative quarks propagator arising from lattice data has already been proposed in the reference [9]. The new idea proposed in the present paper is to analyze in which range of the parameters appearing in the propagators, pressure and energy density satisfy some very basic consistency conditions of thermodynamical equilibrium configurations. Such conditions appear in a very natural way in the analysis of any self-gravitating compact object (like quark stars) in an analogous way to the pioneering work [8].

The first consistency condition is actually so obvious that it was not even explicitly enumerated (but of course assumed) in [8] so that we will call it condition zero.

Condition zero: Existence of an equation of state In the standard general relativistic approach to self-gravitating objects, before even beginning to require some consistency conditions on thermodynamical quantities, it is necessary to have an EOS (namely, a functional relation $P=P(e)$ of the pressure in terms of the energy density). If this condition is not satisfied, there would not exist any obvious way to couple nonperturbative quarks and gluons to General Relativity (nor to any reasonable generalization of General Relativity). As there are some arguments supporting the existence of quark stars [6,7], the issue about the possibility to define an EOS even in such extreme conditions is very relevant.

Condition one: Causality As is well known, in order to enforce causality a necessary condition is to impose the requirement that no signal can travel faster than the speed of light. This means that the speed of sound inside a selfgravitating object cannot be superluminal. In terms of the EOS this condition takes the simple form $\frac{\mathrm{d} P}{\mathrm{~d} e} \leq 1$. It is therefore obvious that, without condition zero, it is not even possible to enforce causality.

Condition two: Le Chatelier's Principle This principle states that for any action intended to modify a given equilibrium configuration of a system, the system will react in such a way that it opposes to the change. This principle is actually equivalent to the assumption that there exists a stable equilibrium configuration. For a self-gravitating object this principle implies that there is no spontaneous gravitational collapse. In terms of the EOS, this principle can be stated as $\frac{\mathrm{d} P}{\mathrm{~d} e} \geq 0$. Once again, it is worth noticing here that without condition zero being satisfied, it is not possible to enforce this principle.

The condition that is prone to fail in the context of the non-perturbative quarks propagator (as well as gluons propagator in the next section) is condition zero. Indeed we will show that it exists a narrow range of temperature close to $T=0$ where the pressure is not a strictly monotonous function. Very important, from this point of view, is the fact that the $\zeta$-function regularization provides results in closed analytic form as sums of (very fast convergent) series of suitable Bessel functions (unlike Ref. [9] in which the authors used dimensional regularization). The grand partition function corresponding to the non-perturbative quark propagator in Eqs. (1) and (2) reads (we will follow the notation of [9])

$$
\frac{\log Z(T, \mu)}{2 \beta V N_{c} N_{f}}=\sum_{n=-\infty}^{+\infty} \int \frac{\mathrm{d}^{3} p}{(2 \pi)^{3}} \log \Lambda^{-2}\left(\Omega^{2}(\mu)+\left(\omega_{n}-i \mu\right)^{2}\right),
$$


where in our case $\Lambda$ is a suitable dimensional parameter, $N_{c}=3, N_{f}=6, \omega_{n}=2 \pi(n+1) T$ are the Matsubara frequencies for fermions, and

$$
\Omega^{2}(\mu)=\boldsymbol{p}^{2}+\left(\frac{M_{3}}{\left(\omega_{n}-i \mu\right)^{2}+\boldsymbol{p}^{2}+m^{2}}+m_{0}\right)^{2} .
$$

It is worth to note that usually the grand partition function is written with $\beta^{2}=1 / T^{2}$ instead of our $\Lambda^{-2}$. However, some subtraction must be done also in such a case in order to avoid the infinite part [16]. In our case, splitting our regulator as $\Lambda^{2}=\beta^{2} \mathcal{N}^{2}$ in (4), where $\mathcal{N}$ is a dimensionless constant, we obtain

$$
\begin{aligned}
\frac{\log Z(T, \mu)}{2 \beta V N_{c} N_{f}}= & \sum_{n=-\infty}^{+\infty} \int \frac{\mathrm{d}^{3} p}{(2 \pi)^{3}} \log \beta^{-2}\left(\Omega^{2}(\mu)+\left(\omega_{n}-i \mu\right)^{2}\right) \\
& +\sum_{n=-\infty}^{+\infty} \int \frac{\mathrm{d}^{3} p}{(2 \pi)^{3}} \log \mathcal{N}^{-2}
\end{aligned}
$$

where we can use precisely the last term to absorb the infinity setting $P(T=0, \mu=0)=0$. At the end, this will give us the same result as [9].

One can simplify the above expression (4) as

$$
\begin{aligned}
I^{\left(\alpha_{i}\right)} & =-\lim _{s \rightarrow 0} \frac{\partial}{\partial s}\left\{(\Lambda)^{2 s} \sum_{n=-\infty}^{+\infty} \int \frac{\mathrm{d}^{3} p}{(2 \pi)^{3}}\left(\left[\boldsymbol{p}^{2}+\alpha_{i}^{2}\left(\omega_{n}, \mu\right)\right]\right)^{-s}\right\} \\
& =-\lim _{s \rightarrow 0} \partial_{s}\left\{(\Lambda)^{2 s} \sum_{n=-\infty}^{+\infty} \frac{\Gamma(s-3 / 2)}{8 \pi^{\frac{3}{2}} \Gamma(s)}\left(\alpha_{i}^{2}\left(\omega_{n}, \mu\right)\right)^{\frac{3}{2}-s}\right\},
\end{aligned}
$$

so that we get

$$
I^{\left(\alpha_{i}\right)}=-\lim _{s \rightarrow 0} \partial_{s}\left\{(\Lambda)^{2 s} \sum_{n=-\infty}^{+\infty} \frac{1}{8 \pi^{\frac{3}{2}} \Gamma(s)} \int_{0}^{\infty} \mathrm{d} t e^{-t \alpha_{i}^{2}} t^{s-5 / 2}\right\} .
$$

In order to proceed, it is necessary to have an explicit form for the $\alpha_{i}$, it can be shown that (see Appendix A for details) $\alpha_{i}^{2}=m_{i}^{2}-\left(\mu+i \omega_{n}\right)^{2}, i=(1,2,3)$. Having $\operatorname{Re}\left(m^{2}\right)>0$ (remember the $\alpha_{i}^{2}$ are the opposite of the roots of the numerator) the integral is convergent, thus

$$
\begin{aligned}
I^{\left(\alpha_{i}\right)}= & -\lim _{s \rightarrow 0} \partial_{s}\left\{(\Lambda)^{2 s} \sum_{n=-\infty}^{+\infty} \frac{T^{3-2 s}}{4^{s} \pi^{2 s-\frac{3}{2}} \Gamma(s)}\right. \\
& \left.\times \int_{0}^{\infty} \mathrm{d} y y^{s-5 / 2} e^{-q_{i}^{2} y} \sum_{n=-\infty}^{\infty} e^{-y(n+c)^{2}}\right\}
\end{aligned}
$$

$$
\begin{aligned}
& \frac{\log Z(T, \mu)}{2 \beta V N_{c} N_{f}}=\sum_{n=-\infty}^{+\infty} \int \frac{\mathrm{d}^{3} p}{(2 \pi)^{3}} \ln \Lambda^{-2} \\
& \quad \times\left[\boldsymbol{p}^{2}+\left(\frac{M_{3}}{\left(\omega_{n}-i \mu\right)^{2}+\boldsymbol{p}^{2}+m^{2}}+m_{0}\right)^{2}+\left(\omega_{n}-i \mu\right)^{2}\right] \\
& =\sum_{n=-\infty}^{+\infty} \int \frac{\mathrm{d}^{3} p}{(2 \pi)^{3}} \ln \Lambda^{-2} \\
& \quad \times\left[\frac{\left(\boldsymbol{p}^{2}+\left(\omega_{n}-i \mu\right)^{2}\right)\left(\boldsymbol{p}^{2}+\left(\omega_{n}-i \mu\right)^{2}+m^{2}\right)^{2}+\left(M_{3}+m_{0}\left(\boldsymbol{p}^{2}+\left(\omega_{n}-i \mu\right)^{2}+m^{2}\right)\right)^{2}}{\left(\boldsymbol{p}^{2}+\left(\omega_{n}-i \mu\right)^{2}+m^{2}\right)^{2}}\right] \\
& =\sum_{i=1}^{i=4} c_{i} \sum_{n=-\infty}^{+\infty} \int \frac{\mathrm{d}^{3} p}{(2 \pi)^{3}} \ln \Lambda^{-2}\left[\boldsymbol{p}^{2}+\alpha_{i}^{2}\left(\omega_{n}, \mu\right)\right]=: \sum_{i=1}^{i=4} I^{\left(\alpha_{i}\right)} c_{i},
\end{aligned}
$$

where $\left\{\alpha_{i}^{2}\left(\omega_{n}, \mu\right),(i=1,2,3)\right\}$ are minus the three roots of the numerator, $\alpha_{4}^{2}=\left(\omega_{n}-i \mu\right)^{2}+m^{2},\left\{c_{i}=1,(i=1,2,3)\right\}$ and $c_{4}=-2$. So, everything reduces to finding, for a generic $\alpha_{i}$, the quantity

$$
I^{\left(\alpha_{i}\right)}=\sum_{n=-\infty}^{+\infty} \int \frac{\mathrm{d}^{3} p}{(2 \pi)^{3}} \ln \Lambda^{-2}\left[\boldsymbol{p}^{2}+\alpha_{i}^{2}\left(\omega_{n}, \mu\right)\right] .
$$

Now, using the fact that $\ln \Lambda^{-2}\left[\boldsymbol{p}^{2}+\alpha_{i}^{2}\left(\omega_{n}, \mu\right)\right]=$ $-\lim _{s \rightarrow 0} \frac{\partial}{\partial s} \ln \left(\Lambda^{2}\left[\boldsymbol{p}^{2}+\alpha_{i}^{2}\left(\omega_{n}, \mu\right)\right]\right)^{-s}$ we can write

$$
\begin{aligned}
= & -\lim _{s \rightarrow 0} \partial_{s}\left\{(\Lambda)^{2 s} \frac{T^{3-2 s}}{4^{s} \pi^{2 s-\frac{3}{2}} \Gamma(s)}\right. \\
& \left.\times \int_{0}^{\infty} \mathrm{d} y y^{s-5 / 2} e^{-q_{i}^{2} y} \sqrt{\frac{\pi}{y}} \vartheta_{3}\left(c \pi, e^{-\pi^{2} / y}\right)\right\},
\end{aligned}
$$

with $c=\frac{1}{2}-i \frac{\mu}{2 \pi T}, q_{i}^{2}=\frac{m_{i}^{2}}{4 \pi^{2} T^{2}}$ and $y=4 \pi^{2} T^{2} t$ and $\vartheta_{3}(x, y)$ the Jacobi $\theta$-function. Using the well-known representation for the Jacobi $\theta$-function 
$\vartheta_{3}(x, y)=1+2 \sum_{n=1}^{\infty} y^{n^{2}} \cos 2 n x$,

the integral can be reduced to

$$
\begin{aligned}
I^{\left(\alpha_{i}\right)}= & -\lim _{s \rightarrow 0} \partial_{s}\left\{( \Lambda ) ^ { 2 s } \left[\frac{T^{3-2 s}\left(q_{i}^{2}\right)^{2-s} \Gamma(s-2)}{4^{s} \pi^{2 s-2} \Gamma(s)}\right.\right. \\
& +\frac{T^{3-2 s}\left(q_{i}^{2}\right)^{1-\frac{s}{2}}}{4^{s-1} \pi^{s} \Gamma(s)} \sum_{n=1}^{+\infty} n^{s-2} K_{2-s} \\
& \left.\left.\times\left(2 n \pi \sqrt{q^{2}}\right) \cos \left(n \pi-i \frac{n \mu}{T}\right)\right]\right\} \\
= & \frac{\left(m_{i}^{2}\right)^{2}}{32 \pi^{2} T}\left(\log \left(\frac{m_{i}^{2}}{\Lambda^{2}}\right)-\frac{3}{2}\right) \\
& +\sum_{n=1}^{+\infty} \frac{m_{i}^{2}(-1)^{n} T}{\pi^{2} n^{2}} K_{2}\left(n \frac{\sqrt{m_{i}^{2}}}{T}\right) \cosh \left(\frac{\mu n}{T}\right),
\end{aligned}
$$

where we assume $\mu<\operatorname{Re}\left(m_{i}\right)$.

\subsection{Existence of equation of state}

From the partition function, we can obtain the pressure, entropy density, number density, and energy density, respectively:

$$
\begin{aligned}
P(T, \mu) & =\frac{T}{V} \log Z(T, \mu), \\
s(T, \mu) & =\frac{\partial P}{\partial T}(T, \mu), \\
n(T, \mu) & =\frac{\partial P}{\partial \mu}(T, \mu), \\
e(T, \mu) & =T s-P+\mu n .
\end{aligned}
$$

Inverting the last equation, we can obtain the EOS either for $\mu$ constant $P=\left.P(e, T)\right|_{\mu}$ or $T$ constant $P=\left.P(e, \mu)\right|_{T}$. We stress here the fact that, if the pressure and energy density function in (10) are not strictly monotonic functions in some interval $J \in \mathbb{R}$ either for $T$ or $\mu$, and also the shape of nonmonotonicity in $J$ does not coincide exactly for $P$ and $e$, then it is not possible to define a proper EOS as a function $P=P(e)$ in the interval $J$.

As is shown in Fig. 1, using the mass fit parameters (2) for the quark propagator, we see there is a range of $T \in(0.0,3.36) \times 10^{-2} \mathrm{GeV}$, which we will call critical zone, where we cannot find an EOS. This is because when we have imaginary a component of the poles of the propagator, the modified Bessel functions $K_{v}$ acquire, below $T=0.036 \mathrm{GeV}$, an oscillatory behavior; see Appendix B. In our opinion, these results suggest that, at low temperature, some extra physical parameters (which, for example, can be related to light glueballs) suitable to properly label the equilibrium states are needed (see Appendix B for some

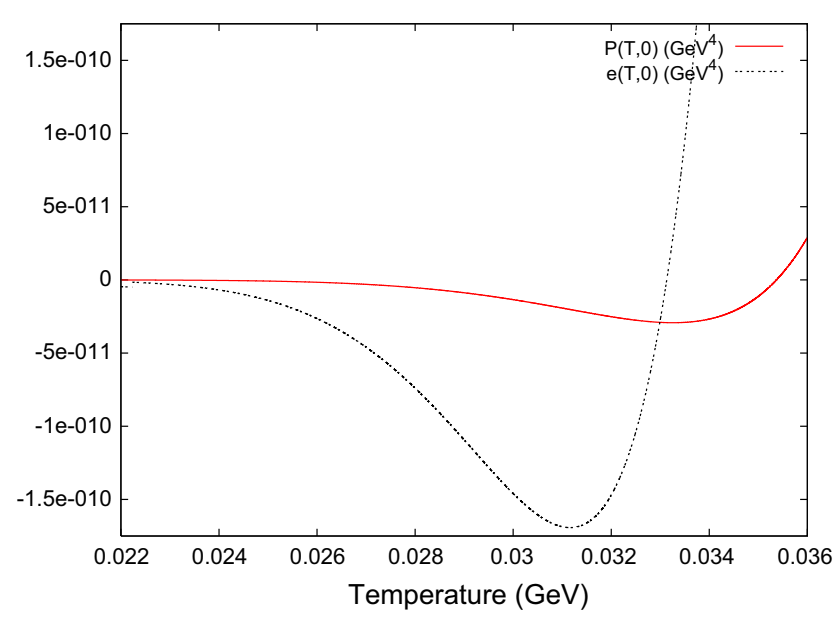

Fig. 1 The pressure $P$ of the quark sector (red line) and its energy density $e$ (black dots) as a function of the temperature for $\mu=0$. It is worth to note the detail of the plot due the critical zone is very narrow compared to the entire unit range and the values of negative $P$ is less than $2 \times 10^{-5} \mathrm{GeV}$

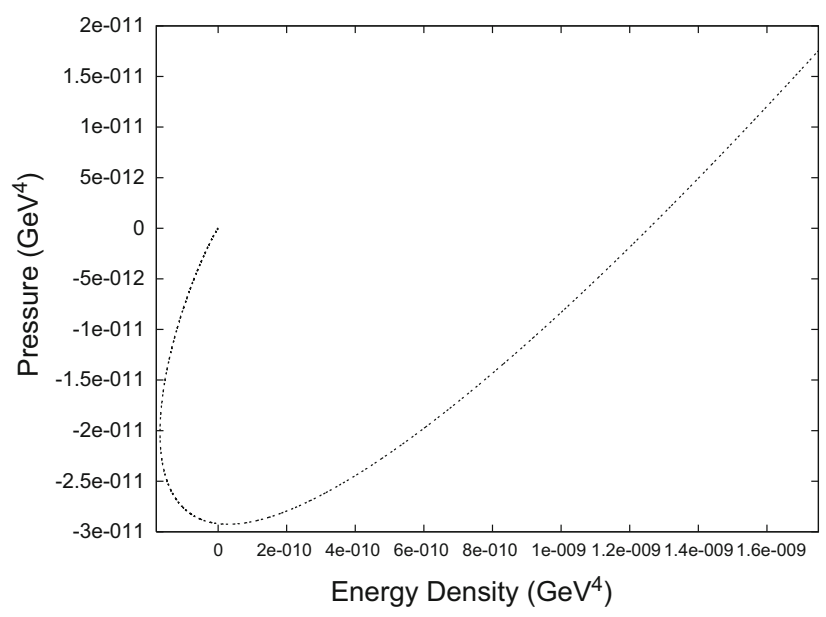

Fig. 2 The pressure $P$ of the quark sector as a function of the energy density $e$ for $\mu=0$. We can see if $e \leq 0$ then the EOS cannot be defined

considerations on this respect). For instance, there is not a unique value of the normalized pressure for a normalized energy density of $-5 \times 10^{-11}$. It is interesting to address the plot of pressure $P$ as a function of the energy density $e$. As is shown in Fig. 2, there is an EOS when both $e$ and $P$ are positive. On the other hand, if we change the fit parameters $+10 \%$, such critical zone would disappear (or it could even increase if we decrease the lattice parameters by $-10 \%$ ), as we can compare in Fig. 3.

\subsection{Checking causality and Le Chatelier's principles}

Once we can write the EOS, we may wonder if the conditions of causality $\left(c^{2}=\frac{\partial P}{\partial e} \leq 1\right)$ and Le Chatelier's principle ( $P$ is monotonically growing with respect to $e$ ), which appearing in 


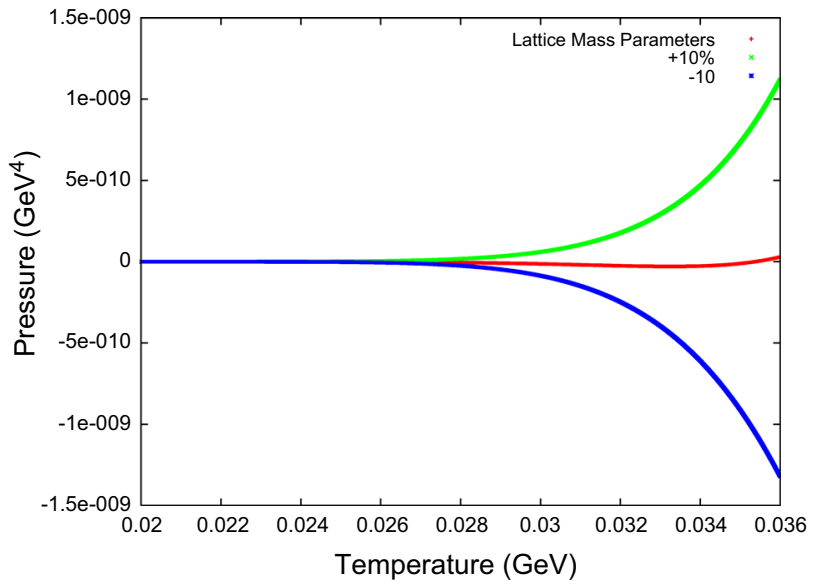

Fig. 3 Comparison of the pressure of the quark sector as a function of temperature in the critical zone for $\mu=0, M_{3}, m^{2}$ and $m_{0}$ given in (2), and $\mathrm{a} \pm 10 \%$ modification of these lattice mass parameters

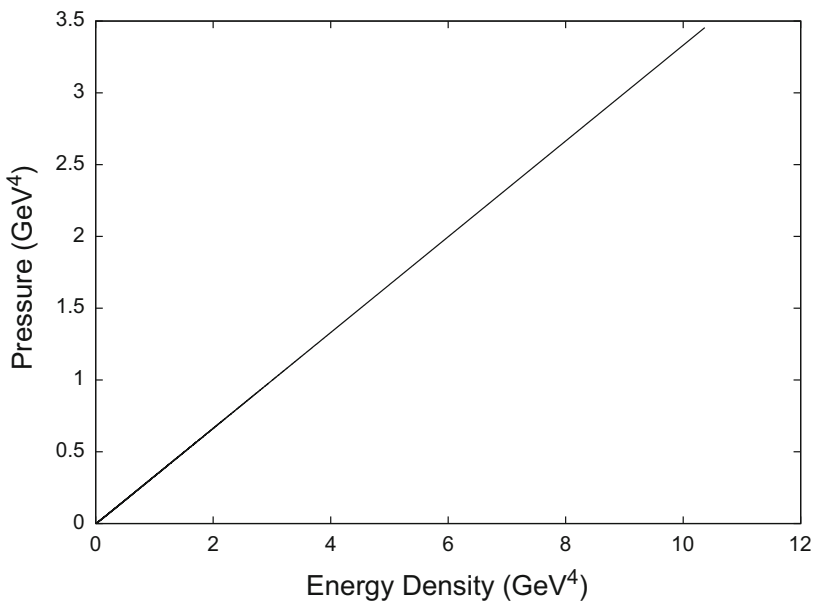

Fig. 4 The pressure $P$ of the quark sector as a function of the energy $e$ for $\mu=0$

[8], are both satisfied. As we can see in Fig. 4, Le Chatelier's principle holds for the mass values of the lattice data, when it is possible to define an EOS $P=P(e)$ (see description above). In Fig. 5, one can see that causality holds as well.

\section{The gluonic sector}

In this section we will check, as we did in the previous section for the quarks, whether or not the non-perturbative gluons propagator in the Landau gauge arising from the lattice [17] gives rise to an EOS satisfying the three principle mentioned above. The extra benefit of this analysis is that, in the gluons case, the non-perturbative propagator arising from the lattice data can also be deduced theoretically. Such a propagator is strongly related with one of the most fascinating non-perturbative effects in non-Abelian gauge theories: the

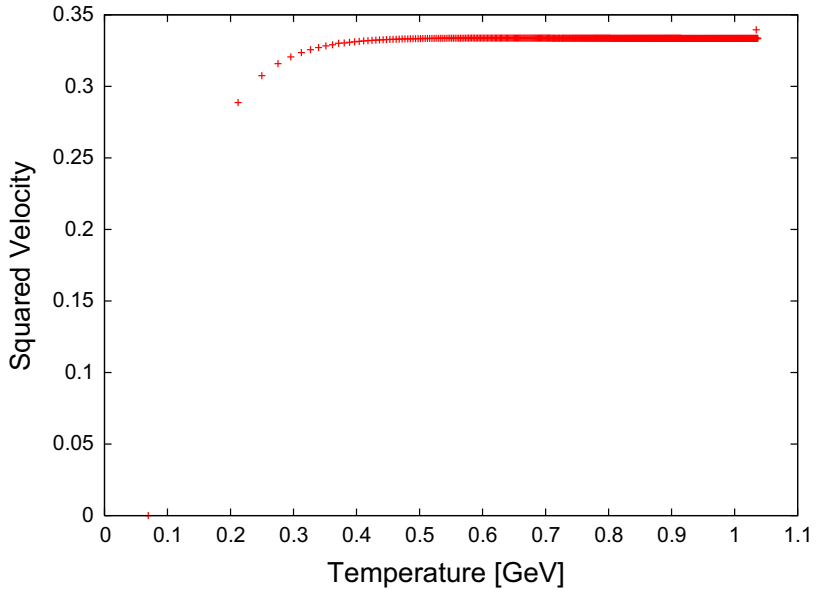

Fig. 5 The squared sound velocity of the quark sector as a function of the temperature for $\mu=0$

appearance of Gribov copies [2]. On flat, topologically trivial, space-times (which is the only case which will be analyzed here) Gribov copies represent a topological obstruction to define globally the gauge-fixing peculiar of the non-Abelian nature of the gauge group (for a good review see [18]).

On the other hand, both on curved spaces and on flat spaces but with non-trivial topology the pattern of appearance of Gribov copies can be considerably more complicated. For instance, Gribov copies can appear even in the Abelian case (see in particular [19-25]). The results in these references suggest, as a future direction, to analyze how the equations of state of interacting gauge bosons (even in the Abelian case) depend on the non-trivial backgrounds on which they are defined. We hope to come back on this interesting issue in a future publication.

The most effective method to eliminate Gribov copies (proposed by Gribov himself in [2] and refined in [26-28]) amounts to restrict the domain of the path integral to the region in which the Faddeev-Popov operator is positive definite (such region is called Gribov region). In [29] the authors showed that all the gauge orbits cross the Gribov region. Hence, the GZ restriction does not lose any relevant physical configuration. A local and renormalizable effective action for Yang-Mills theory whose dynamics is restricted to the Gribov horizon was constructed in [30-33] by adding extra fields to the action. Later, an improved action was proposed by considering suitable condensates [34], which leads to propagators and glueball masses in agreement with the lattice data [35]. With the same action, one can also solve the old problem of the Casimir energy in the MIT-bag model [36]. Moreover, this approach is quite effective also at finite temperature [37-39] and, at least at one-loop order, gives rise to a vacuum expectation value for the Polyakov loop compatible with its role of order parameter for the confinement-deconfinement transition [40]. 


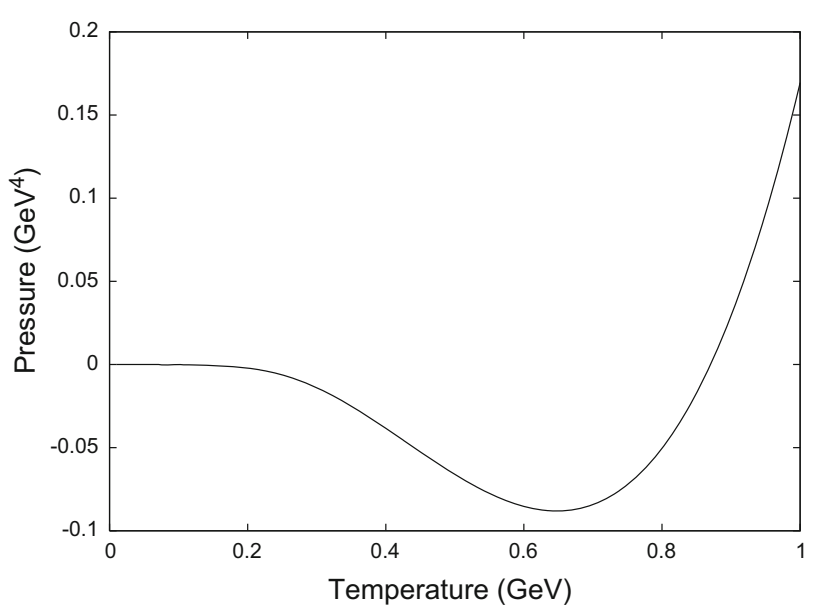

Fig. 6 The pressure $P$ as a function of the temperature $T$ in GZ approach. It can be seen a range of $T$ where $P$ is negative

\subsection{Gribov-Zwanziger approach}

Within the GZ approach, the vacuum energy density at one loop can be written as (we will follow the notation of [40]):

$$
\begin{aligned}
\mathcal{E}_{v}= & -\frac{d\left(N^{2}-1\right)}{2 N g^{2}} \lambda^{4}+\frac{1}{2 \beta V}(d-1) \ln \left(\frac{D^{4}+\lambda^{4}}{\Lambda^{4}}\right) \\
& -\frac{d}{2 \beta V} \ln \left(\frac{-D^{2}}{\Lambda^{2}}\right)
\end{aligned}
$$

being $\Lambda$ a regularization parameter, $\beta=1 / T$ and $V$ the spatial volume. One can normalize the Gribov parameter $\lambda$ in order to absorb the divergent part of (11). In this way one obtains for the $S U(2)$ internal gauge group

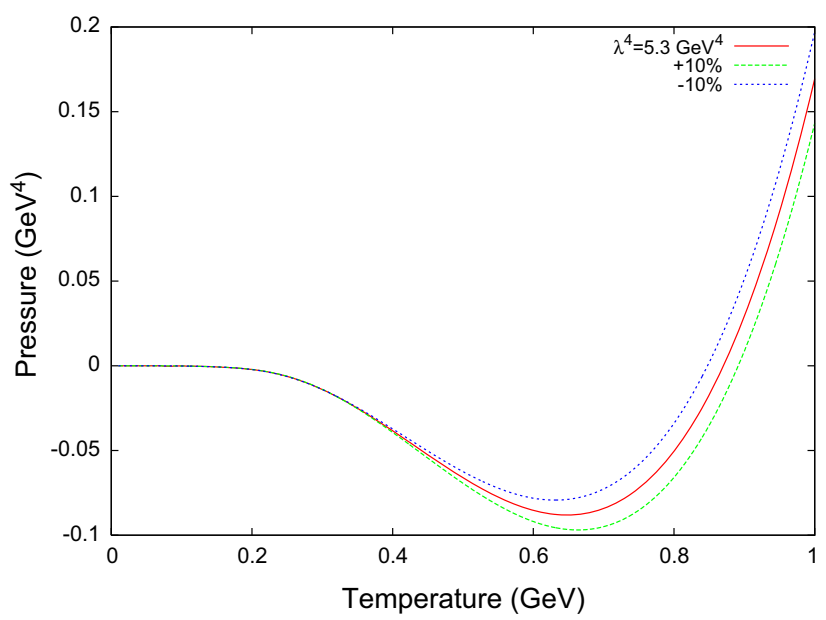

(a)
$\mathcal{E}_{v}(T)=\frac{3}{2}(d-1)\left[I\left(T, i \lambda^{2}\right)+I\left(T,-i \lambda^{2}\right)\right]-\frac{d}{2} I(T, 0)$,

where in the $d=4$ case

$$
\begin{aligned}
I\left(T, \alpha^{2}\right)= & T \sum_{n=-\infty}^{+\infty} \int \frac{\mathrm{d} p^{3}}{(2 \pi)^{3}} \ln \Lambda^{2}\left(\omega_{n}^{2}+m^{2}+\mathbf{p}^{2}\right) \\
= & \frac{\left(\alpha^{2}\right)^{2}}{32 \pi^{2}}\left(\ln \left(\frac{\alpha^{2}}{\Lambda^{2}}\right)-\frac{3}{2}\right) \\
& -\frac{\alpha^{2} T^{2}}{\pi^{2}} \sum_{n=1}^{+\infty}(-1) n^{-2} K_{2}\left(n \frac{m}{T}\right),
\end{aligned}
$$

where we have taken the thermodynamic limit $V \rightarrow+\infty$ in the second equality, which implies $\sum_{q} \rightarrow V \int \frac{\mathrm{d}^{3} q}{(2 \pi)^{3}}$ [18]. The detailed computations of (13) are in Appendix A.

In principle, as we are working at finite temperature, one should first determine how the Gribov mass parameter $\lambda$ depends on the temperature itself (see [37-39]). However, this would lead to a coupled system of integral equations to be solved self-consistently and this would enormously complicate the numerical task. Fortunately, as the available results clearly indicate, the Gribov parameter $\lambda$ changes very slowly in the range of temperatures analyzed in the present paper (see Figure 2 of [40] where $\lambda$ was computed as a function of $T$, the only reasonable assumption being that the coupling constant $g$ does not change in a small range around $T=0$ ) so that we will work in the approximation in which $\lambda$ does not depend on the temperature and is actually equal to its $T=0$ value (see [41], where $\lambda^{4}=5.3 \mathrm{GeV}^{4}$ ). In such a case, we can construct $P(T)=-\mathcal{E}_{v}(T)$ and the result is plotted in Fig. 6. Interestingly, as was also suggested by the results on the Polyakov loop [40], there is a range of temperatures where the pressure is negative and is not a strictly monotonic

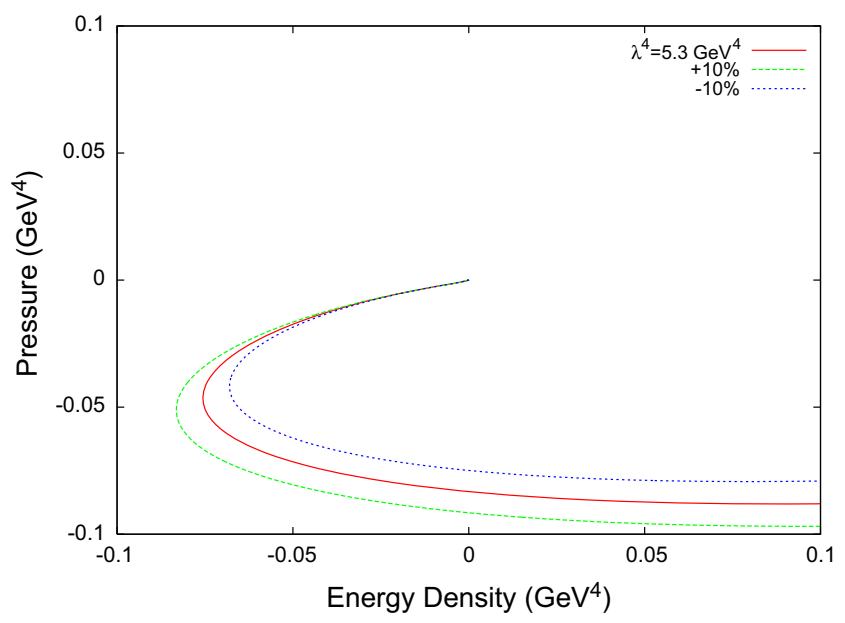

(b)

Fig. 7 a The gluon pressure as a function on temperature for different values of the Gribov parameter $\lambda$ in GZ approach. b Pressure as a function of energy density for different values of the Gribov parameter $\lambda$ in GZ approach 


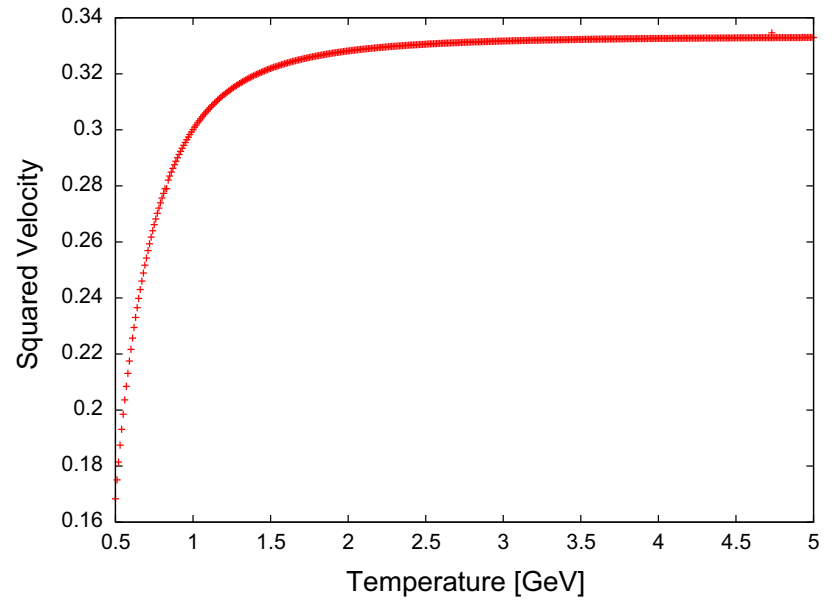

Fig. 8 The squared velocity as a function of the temperature in GZ approach

function. Furthermore, if we modify the Gribov parameter by \pm the $10 \%$ of its value, the plot remains almost the same, as we can see in Fig. 7. Namely, within the GZ approach, the zeroth consistency condition of [8] is likely to be always violated.

In Fig. 8 we plotted the squared velocity as a function of temperature in the region where we can define an EOS. We see Le Chatelier's principle and causality conditions are both satisfied.

It is interesting to show if the zeroth consistency condition is fulfilled in dimensions $d=2$ and $d=3$ also with $S U(2)$ internal gauge group. The computation could be done using (12) and considering ${ }^{1}$ for $d=2$

$I\left(T, \alpha^{2}\right)=-\frac{2}{\pi} T \sqrt{\alpha^{2}} \sum_{n=1}^{+\infty} n^{-1} K_{1}\left(n \frac{\sqrt{\alpha^{2}}}{T}\right)$,

while for $d=3$

$I\left(T, \alpha^{2}\right)=-\frac{\sqrt{2}}{\pi^{3 / 2}} T^{3 / 2}\left(\alpha^{2}\right)^{3 / 4} \sum_{n=1}^{+\infty} n^{-3 / 3} K_{3 / 2}\left(n \frac{\sqrt{\alpha^{2}}}{T}\right)$,

and using for both cases the Gribov parameter $\lambda^{4}$ given in [41]. As is shown in Fig. 9, both in $d=2$ and $d=3$, there is a region where the EOS cannot be well defined in the GZ approach.

\subsection{Refined Gribov-Zwanziger approach}

In this subsection, we consider the non-perturbative gluon EOS taking into account the appearance of the condensates in the propagator [34] which is favored by lattice data [17]

\footnotetext{
$\overline{1}$ We take only the modes $n \neq 0$ for $I\left(T, \alpha^{2}\right)$ in dimensions $d=2$ and $d=3$.
}

(following the same technique to compute the partition function (4)). The RGZ-propagator is [41]

$$
\begin{aligned}
\Delta_{\mu \nu}^{a b}(p) & =\delta^{a b} \frac{p^{2}+N^{2}}{p^{4}+p^{2}\left(N^{2}+m^{2}\right)+m^{2} N^{2}+\lambda^{4}}\left(\delta_{\mu \nu}-\frac{p_{\mu} p_{v}}{p^{2}}\right) \\
& =\delta^{a b} \Delta\left(p^{2}\right)\left(\delta_{\mu \nu}-\frac{p_{\mu} p_{\nu}}{p^{2}}\right),
\end{aligned}
$$

$N^{2}, m^{2}$ being condensate-related values, and $\lambda$ the Gribov mass parameter. In this case the renormalized RGZ-vacuum energy at one loop can be written as [40]

$$
\begin{aligned}
\mathcal{E}_{v}(T)= & \frac{3}{2}(d-1) \sum_{s=-1}^{s=1}\left[I\left(T, r_{+}^{2}\right)+I\left(T, r_{+}^{2}\right)-I\left(T, N^{2}\right)\right] \\
& -\frac{3}{2} I(T, 0),
\end{aligned}
$$

where the function $I$ is the same as (13) and $r_{ \pm}^{2}$ are the minus roots of the denominator of the RGZ-propagator, which are given by

$r_{ \pm}^{2}=\frac{\left(m^{2}+N^{2}\right) \pm \sqrt{\left(m^{2}+N^{2}\right)^{2}-4\left(m^{2} N^{2}+\lambda^{4}\right)}}{2}$,

where the condensate values $m^{2}, N^{2}$ and the Gribov parameter $\lambda$ were extracted from [41]:

$N^{2}=2.51 \mathrm{GeV}^{2}$,

$m^{2}=-1.92 \mathrm{GeV}^{2}$,

$\lambda^{4}=5.3 \mathrm{GeV}^{4}$.

In Fig. 10 are plotted the pressure $P$ (red line) and the energy density (black dots) as functions of temperature $T$. Again a region is observed where the EOS is not well defined. However, in this case if we change the fit parameters $\pm 10 \%$, then the result is drastically different. In fact, as we show in Fig. 11a, for a modification $+10 \%$ of the RGZ-parameters, the critical region reduces considerably. Also, from Fig. 11b, we can infer that the EOS can be defined for $e \geq 0$ and for almost all $T$ for a modification $+10 \%$ of the RGZparameters. In order to see how the modified parameters change the behavior of the propagator with respect to the original values, we plot in Fig. 12 the function $\Delta\left(p^{2}\right)$ defined in (16) for different values of the condensates parameters $N^{2}$, $m^{2}$ and Gribov mass parameter $\lambda$. We can see that the curves are significantly modified for changes of 5 and $10 \%$ of these parameters. Even more, for a change of $10 \%$ (either in the condensates or Gribov mass parameter) the real poles in the positive axis generate negatives values of $\Delta\left(p^{2}\right)$. This means that even if by changing the parameters it is possible fix the thermodynamic problem, the corresponding propagators are quite off-scale ${ }^{2}$ with respect to the lattice results.

\footnotetext{
$\overline{2}$ In particular, an intuitive way to realize the lattice propagator is very different from the propagator with real poles (without thermodynamics
} 


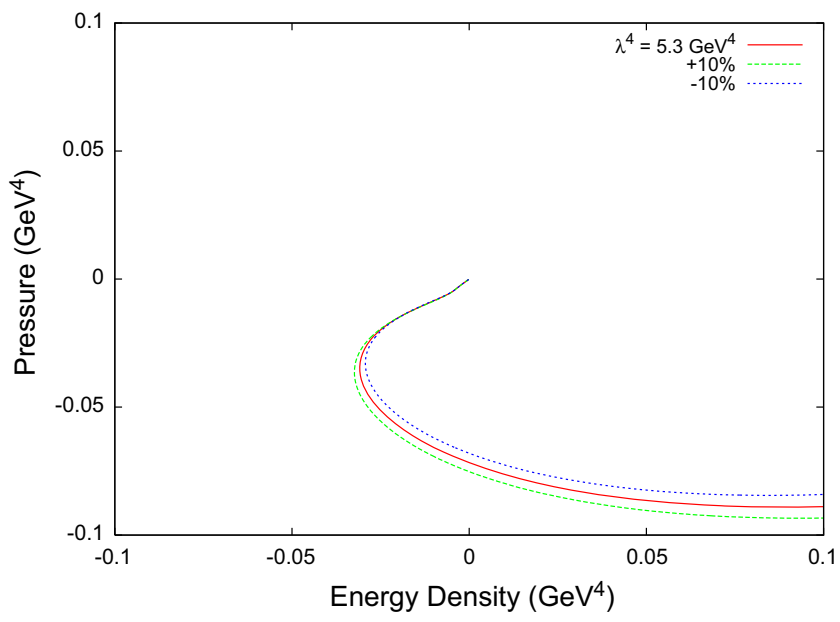

(a)

Fig. 9 a Pressure as a function of energy density and different values of the Gribov parameter $\lambda$ in GZ approach in dimension $d=2$. We take the $\lambda^{4}$ value from [41]. b Pressure as a function of energy density and

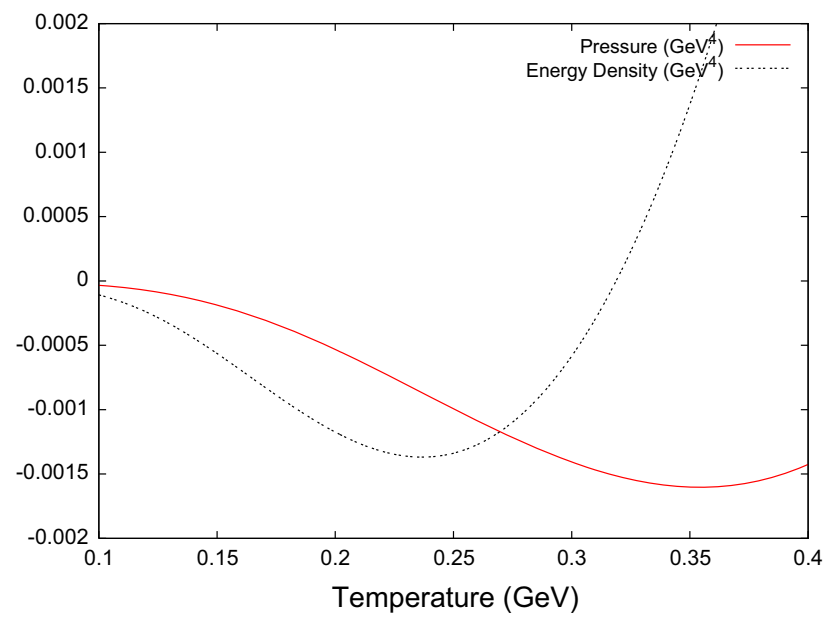

Fig. 10 The gluon pressure (red line) and gluon energy density (black dots) as functions of temperature for $\mu=0$ in the RGZ-approach

Taking into account that in dimension $d=3$ there exists also the possibility to refine the GZ approach [42], which is not the case for $d=2$ [43], we can perform the same analysis in three dimensions and the results are qualitatively the same as in dimension $d=4$.

\section{Conclusions and perspectives}

In this paper, the existence and properties of the EOS derived from the non-perturbative quark and gluon propagators are

\section{Footnote 2 continued}

pathologies) is due to the difference of the discriminants, which determines whether the poles are real or complex conjugated. Such a distance is at least as big as the absolute value of the discriminant of the lattice propagator itself, which is around $1.6 \mathrm{GeV}^{4}$ for the lattice values (19).

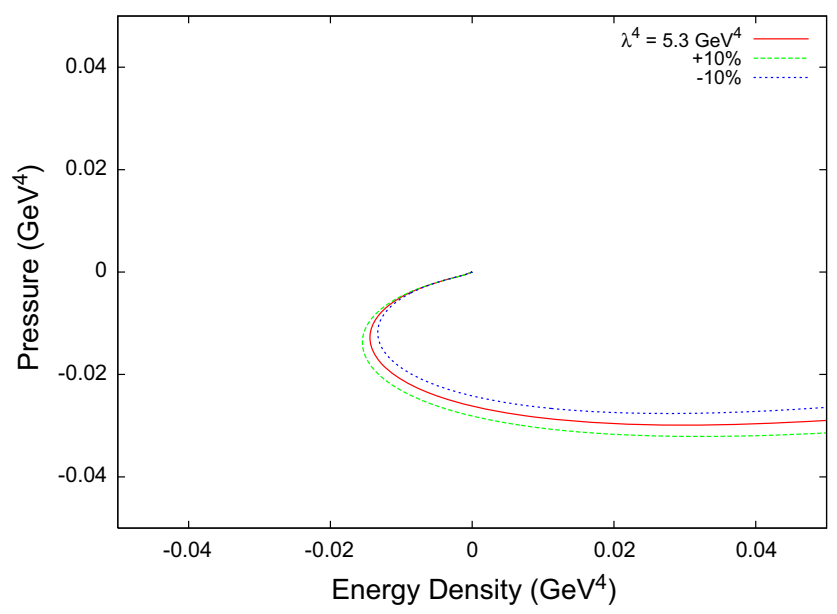

(b)

different values of the Gribov parameter $\lambda$ in GZ approach in dimension $d=3$. We take the $\lambda^{4}$ value from [41]

analyzed at one loop. In order to reduce as much as possible the number of numerical integrations (and, correspondingly, the numerical error) the $\zeta$-function regularization method is used. The present computations are compatible with previous results found in Ref. [9]. However, with the $\zeta$-function regularization method it is possible to disclose very tiny effects that, otherwise, are difficult to disentangle from the numerical errors. Indeed, in the case of quarks, the analysis reveals that, depending on the exact value of the parameters in the fit of the lattice quark propagator, the pressure as a function of the temperature (at fixed chemical potential) can be a non-monotonic function of the temperature.

Even if the effect is small, the non-monotonic behavior of the pressure in this temperature interval implies that the pressure as a function of the energy density is not single valued (namely, one cannot define an EOS in the usual sense). By changing the fit parameters of at least $+10 \%$, this feature almost disappears. The physical explanation (see Appendix B) of this result is the following: the nonperturbative propagators analyzed in the present manuscript can have both complex conjugated poles (as suggested by the lattice data) and real poles. In both cases such propagators violate positivity (in the former case due to the complex conjugated square masses, in the latter case due to the fact that there is always a negative residue) and so they describe confined degrees of freedom. It is true that complex conjugated poles are more natural to describe confined degrees of freedom (as first recognized by Gribov and Zwanziger) and are also supported by lattice data. However, it is interesting to explore what happens when, instead of a pair of complex conjugated poles, one gets two real poles with opposite residues. In this case, as we have explicitly shown in this manuscript, there is no pathological behavior in the thermo- 


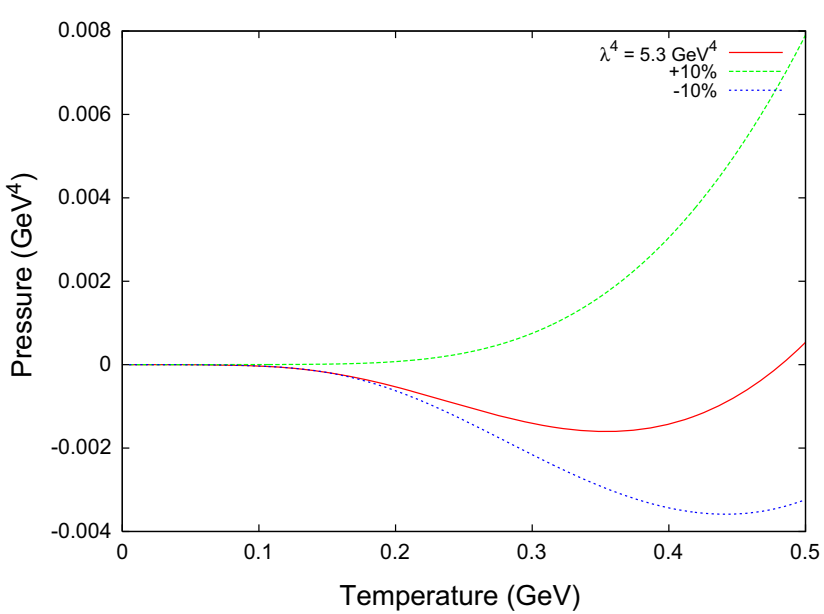

(a)

Fig. 11 a The pressure as a function of temperature for $\mu=0$ and different values of the lattice mass parameters in the RGZ-approach. We see in this critical region, the EOS is not well defined. $\mathbf{b}$ The pressure

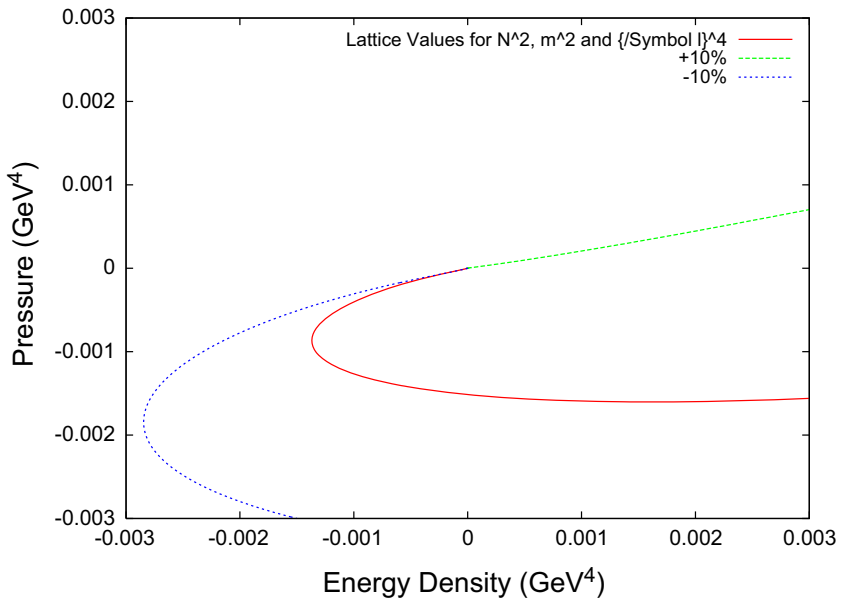

(b)

as a function of the energy density for different values of the RGZparameters $N^{2}, m^{2}$ and $\lambda^{4}$. We observe that an EOS seems to be almost well defined when the RGZ-parameters are modified $+10 \%$

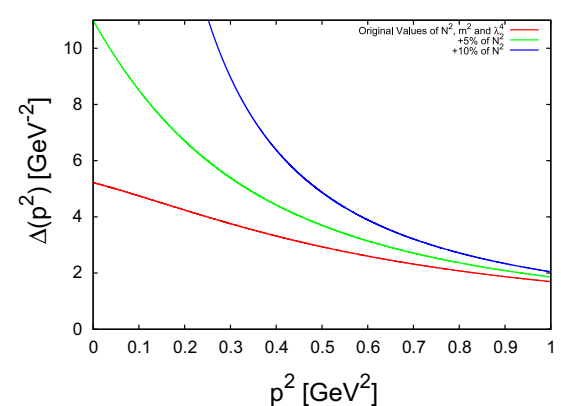

(a)

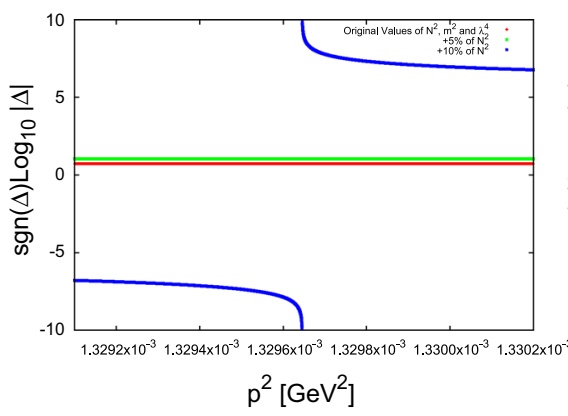

(d)

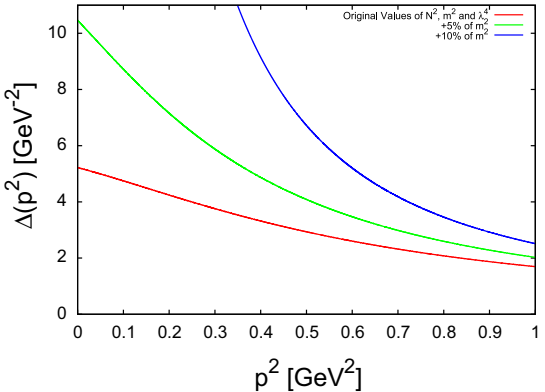

(b)

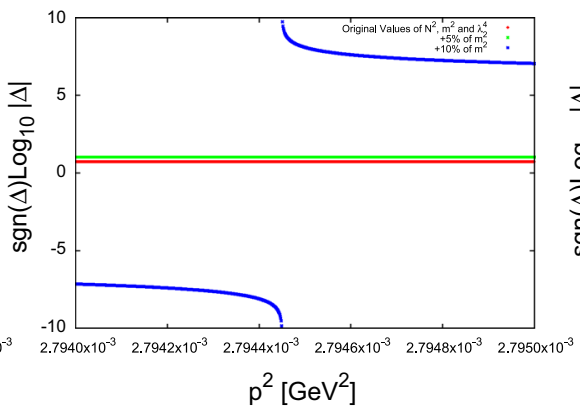

(e)

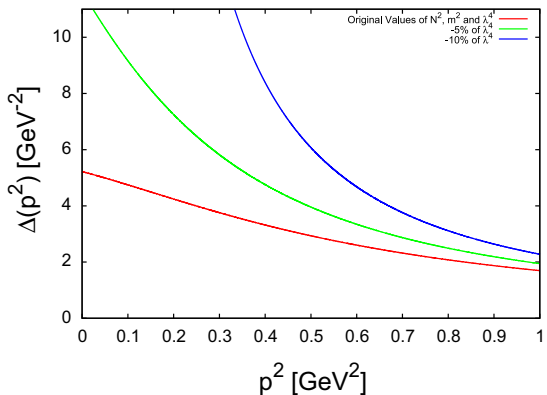

(c)

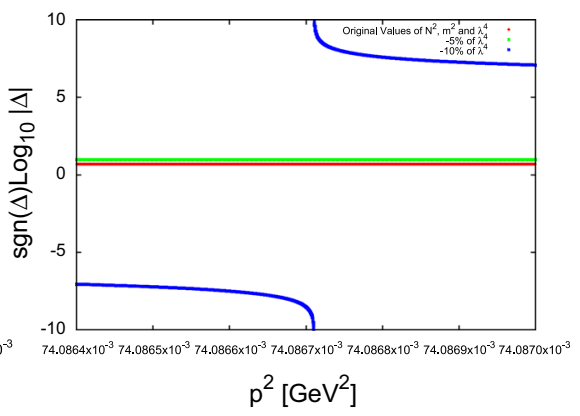

(f)
Fig. 12 Above Comparison of the curve $\Delta\left(p^{2}\right)$ in the unit range of square momenta $p^{2}$ for the original mass lattice values (red line) with respect to a modifying only $N^{2}$ in $+5 \%$ (green line) and $+10 \%$ (blue line); $\mathbf{b}$ modifying only $\mathrm{m}^{2}$ in $+5 \%$ (green line) and $+10 \%$ (blue line); modifying only $\lambda^{4}$ in $-5 \%$ (green line) and $-10 \%$ (blue line). Below To see more clearly how the modified propagator is out of scale with respect to the lattice values, we plot $\operatorname{sgn}(\Delta) \log _{10} \Delta$ in the square momenta

dynamics (despite the violation of positivity). The price to pay is, obviously, that it is not easy to interpret a propagator with two real poles which can be split into two terms (one range close to the real poles which appear $\mathbf{d}$ in $p^{2} \approx 1.3 \times 10^{-3} \mathrm{GeV}^{2}$ when only $N^{2}$ is modified $+10 \%$; $\mathbf{e}$ in $p^{2} \approx 2.8 \times 10^{-3} \mathrm{GeV}^{2}$ when only $m^{2}$ is modified $+10 \%$; $\mathbf{f}$ in $p^{2} \approx 7.4 \times 10^{-2} \mathrm{GeV}^{2}$ when only $\lambda^{4}$ is modified $-10 \%$. Observe also how, since the poles are in the real positive axis in the modified propagators, the function $\Delta\left(p^{2}\right)$ acquires negatives values

with positive and one with negative residue). Such behavior can appear, for instance, when considering the semi-classical Gribov approach at finite temperature [38]. Our personal 
opinion is that complex conjugated poles are clearly favored and, correspondingly, the pathologies in the thermodynamical behavior which are observed are related to the fact that some degrees of freedom (such as light glueballs) - which, unfortunately, are difficult to describe analytically - are missing at low temperatures. Nevertheless, we think that it is an interesting observation that one can both keep alive the violation of positivity and, at the same time, solve the thermodynamical pathologies mentioned above by using real poles with opposite residues. For the sake of clarity, in Appendix B we present a concrete example of how the propagator changes when one moves to real poles.

The same analysis has been performed for the nonperturbative gluon and ghost propagators in the GZ parametrization, and the results are very similar. Moreover, in this case, the present analysis is able to clearly distinguish between the scenarios with and without condensates. The reason is that, if one insists on extracting an EOS in the usual sense, then it is necessary to use the refined version which includes the condensates (and which is favored by lattice data). Otherwise, in the case in which the only non-perturbative parameter is the Gribov mass, not even a $10 \%$ change in its value can guarantee the existence of an EOS. The reason of this could be traced on the fact that it is not possible to avoid complex poles in the gluon propagator changing the Gribov parameter $\lambda^{4}$ for the GZ approach. This is not the case for the RGZ-approach, where one has more parameters to play with and for which could be, in principle, obtained real poles (see Appendix B for details). However, even in the case of real poles, one of the residues of the RGZ-propagator has negative sign, violating positivity of the Källén-Lehmann spectrum representation, indicating still some sort of confinement (see some examples of this in $[38,44,45])$.

Our interpretation of the present results (both in the quarks case and in the gluon case) is not that it is mandatory to require the fulfillment of the three requirements mentioned in the previous sections. Rather, it is that strong quantum effects could be able to violate (the first of) the three conditions (the zeroth requirement of [8] mentioned in the previous sections). At least in the cases analyzed here, once the condition to have a well-defined EOS is satisfied, the causality and Le Chatelier principles are both satisfied. However, the first condition can be violated, although in small intervals, and this can have a rather big impact on the physical applications of the non-perturbative equations of state of quarks and gluons. In particular, the Rhoades-Ruffini bound would not be applicable anymore and one could have quark stars more massive than expected. Due to the fact that already a relatively small change in the fit parameters of the lattice propagators can enforce the consistency conditions of selfgravitating QCD matter, it is of course of interest to explore the phenomenological consequences of modifications of such parameters using the mentioned consistency conditions as guideline.

Still it is possible that the thermodynamics at two-loop computations could have better behavior for the pressure and entropy as is the case for the massive Landau-DeWitt action [46]. However, as for the (R)GZ-approach higher loops computations are very difficult, it is not easy to see what happens in the gluonic sector of this model. Nevertheless, we tend to believe this behavior of the thermodynamical quantities is not just a technical issue but it is related to the lack of degrees of freedom at low temperatures (confined phase). After all, we are using propagators which contain a lot of non-perturbative information (as they come from very precise lattice fits). It is worth emphasizing that the existence of the EOS is an assumption of great importance in the astrophysical context. For instance, a great part of the theory of gravitational hydrostatic equilibrium is based on the assumption that there is a well-defined functional relation between pressure and energy density. As there is also evidence supporting the existence of quark stars (see the review [6,7]), it is clear that the present results can be quite relevant in applications.

On the other hand, it is a very concrete possibility that the future lattice data will confirm the actual values of the fit parameters of the lattice propagators and from an analytical point of view the thermodynamics quantities have the same behavior at two or more loops. Indeed, we have also shown that the propagators with real poles (which are free from thermodynamical pathologies) are quite off-scale with respect to the lattice data. Thus, it is unlikely that higher loops effects can take care of such a difference. In such a case, an EOS (at least in the usual sense) would be unavailable. A nonsingle-valued relation between pressure and energy density suggests that there is some information missing when considering strongly interacting quarks and/or gluons. In other words, in such situations, some extra physical parameters able to properly label the equilibrium states are needed (see Appendix B). This, of course, is a quite interesting conclusion. We think that all these issues are worth to be further investigated in the future.

Acknowledgments We would like to thanks D. Dudal, M. Guimaraes, B. Mintz, L. F. Palhares, S. P. Sorella, M. Tissier and N. Wschebor for enlightening discussions and very useful suggestions. P. P. thanks the Universidade do Estado do Rio de Janeiro (UERJ) for the kind hospitality during the development of this work. This work is supported by Fondecyt Grants 1160137, 1150246 and 1160423 . P. P. was partially supported from Fondecyt Grant 1140155 . The Centro de Estudios Científicos (CECS) is funded by the Chilean Government through the Centers of Excellence Base Financing Program of Conicyt. A. Z. is also partially supported by Proyecto Anillo ACT1406.

Open Access This article is distributed under the terms of the Creative Commons Attribution 4.0 International License (http://creativecomm ons.org/licenses/by/4.0/), which permits unrestricted use, distribution, and reproduction in any medium, provided you give appropriate credit to the original author(s) and the source, provide a link to the Creative 
Commons license, and indicate if changes were made. Funded by SCOAP ${ }^{3}$.

\section{Appendix A: Detailed computation of $I\left(T, \mu, \alpha^{2}\right)$}

We saw in the paper that to have a manageable expression of $I\left(T, \mu, \alpha^{2}\right)$ is very important for the quark and gluonic sector, so we include in this appendix the detailed computation of this quantity using $\zeta$-functions in the fermionic and bosonic case. ${ }^{3}$ The definition of the generic $I$ function is

$$
\begin{aligned}
I\left(T, \mu, \alpha^{2}\right)= & \sum_{n=-\infty}^{+\infty} \int \frac{\mathrm{d}^{3} p}{(2 \pi)^{3}} \ln \Lambda^{-2} \\
& \times\left[p^{2}+\left(\omega_{n}-i \mu\right)^{2}+\alpha^{2}\right],
\end{aligned}
$$

where $\omega_{n}$ are the Matsubara frequencies $(2 \pi n T$ in the bosonic case and $2 \pi(n+1) T$ in the fermionic case $), \Lambda$ is a free parameter which we use to regularize, and $\alpha^{2}$ is a mass parameter which we allow to acquire a complex value. We can write $I$ as the derivative with respect to some auxiliary variable $s$ and then taking the limit $s \rightarrow 0$ :

$$
\begin{aligned}
I= & \lim _{s \rightarrow 0} \frac{\partial}{\partial s}\left(-T \Lambda^{2 s} \sum_{n=-\infty}^{+\infty} \int \frac{\mathrm{d}^{3} q}{(2 \pi)^{3}}\right. \\
& \left.\times\left(\left(\omega_{n}-i \mu\right)^{2}+\alpha^{2}+\mathbf{q}^{2}\right)^{-s}\right) .
\end{aligned}
$$

Defining a new variable $t$ as $|\mathbf{q}|=t \sqrt{\left(\omega_{n}-i \mu\right)^{2}+\alpha^{2}}$ and passing to spherical coordinates, we have

$$
\begin{aligned}
I= & \lim _{s \rightarrow 0} \frac{\partial}{\partial s}\left(-T \Lambda^{2 s} \frac{4 \pi}{(2 \pi)^{3}} \sum_{n=-\infty}^{+\infty}\left(\left(\omega_{n}-i \mu\right)^{2}+\alpha^{2}\right)^{\frac{3}{2}-s}\right. \\
& \left.\times \int_{0}^{+\infty} \mathrm{d} t t^{2}\left(1+t^{2}\right)^{-s}\right) .
\end{aligned}
$$

Let us focus on the last integral. We can write it as

$$
\int_{0}^{+\infty} \mathrm{d} t t^{2}\left(1+t^{2}\right)^{-s}=\frac{1}{4} \sqrt{\pi} \frac{\Gamma\left(s-\frac{3}{2}\right)}{\Gamma(s)},
$$

where we used the $\Gamma$ property $(s-1) \Gamma(s-1)=\Gamma(s)$. Thus, one gets

$$
\begin{aligned}
I=\lim _{s \rightarrow 0} & \frac{\partial}{\partial s}\left(-T \Lambda^{2 s} \frac{\Gamma\left(s-\frac{3}{2}\right)}{8 \pi^{\frac{3}{2}} \Gamma(s)}\right. \\
& \left.\times \sum_{n=-\infty}^{+\infty}\left(\left(\omega_{n}-i \mu\right)^{2}+\alpha^{2}\right)^{\frac{3}{2}-s}\right) .
\end{aligned}
$$

\footnotetext{
${ }^{3}$ Because the computation for fermions and bosons are very similar, for completeness we keep $\mu$ during the calculation, but at the end only fermions will be considered with chemical potential.
}

Moreover, taking into account the definition of the $\Gamma$ function,

$\Gamma(t)=\int_{0}^{+\infty} x^{t-1} e^{-x} \mathrm{~d} x$

one gets

$$
\begin{gathered}
\Gamma\left(s-\frac{3}{2}\right)\left(\left(\omega_{n}-i \mu\right)^{2}+\alpha^{2}\right)^{\frac{3}{2}-s} \\
=\int_{0}^{+\infty} \mathrm{d} w w^{s-\frac{5}{2}} e^{-w\left(\left(\omega_{n}-i \mu\right)^{2}+\alpha^{2}\right)} .
\end{gathered}
$$

Defining $y=w 4 \pi^{2} T^{2}, v^{2}=\frac{\alpha^{2}}{4 \pi^{2} T^{2}}$, and $c_{\epsilon}=\epsilon / 2-\frac{i \mu}{2 \pi}$, where $\epsilon=1$ for fermions and $\epsilon=0$ for bosons, we arrive at

$$
\begin{aligned}
I= & \lim _{s \rightarrow 0}-\frac{\partial}{\partial s}\left(\frac{\Lambda^{2 s} T^{4-2 s}}{2^{2 s} \pi^{2 s-\frac{3}{2}} \Gamma(s)} \int_{0}^{+\infty} \mathrm{d} y y^{s-\frac{5}{2}} e^{-y v^{2}}\right. \\
& \left.\times \sum_{n=-\infty}^{+\infty} e^{-y\left(n+c_{\epsilon}\right)^{2}}\right) .
\end{aligned}
$$

Let us focus now on the last sum. We can use the Poisson summation formula,

$$
\sum_{n=-\infty}^{+\infty} f(x+n)=\sum_{k=-\infty}^{+\infty} e^{2 \pi i k x} \int_{-\infty}^{+\infty} f\left(x^{\prime-2 \pi i k x^{\prime}} \mathrm{d} x^{\prime} .\right.
$$

In our case $f(x)=e^{-y x^{2}}$ so, completing the square,

$$
\int_{-\infty}^{+\infty} e^{-y x^{\prime 2}} e^{-2 \pi i k x^{\prime}} \mathrm{d} x^{\prime \frac{-k^{2} \pi^{2}}{y}} \int_{-\infty}^{+\infty} e^{-y\left(x^{\prime}+\frac{i k \pi}{y}\right)^{2}} \mathrm{~d} x^{\prime \frac{-k^{2} \pi^{2}}{y}} \sqrt{\frac{\pi}{y}},
$$

where in the last equality we used the result $\int_{-\infty}^{+\infty} e^{-y(x+a)^{2}} \mathrm{~d} x$ $=\sqrt{\frac{\pi}{y}}$. We arrive at

$$
\sum_{n=-\infty}^{+\infty} e^{-y\left(n+c_{\epsilon}\right)^{2}}=\sqrt{\frac{\pi}{y}}\left(1+2 \sum_{n=1}^{+\infty} \cos \left(2 \pi k c_{\epsilon}\right) e^{-\frac{k^{2} \pi^{2}}{y}}\right) .
$$

Now, we have

$$
\begin{aligned}
I= & \lim _{s \rightarrow 0}-\frac{\partial}{\partial s}\left(\frac{\mu^{2 s} T^{4-2 s}}{2^{2 s} \pi^{2 s-\frac{3}{2}} \Gamma(s)} \int_{0}^{+\infty} \mathrm{d} y y^{s-\frac{5}{2}} e^{-y v^{2}} \sqrt{\frac{\pi}{y}}\right. \\
& \left.\times\left(1+2 \sum_{n=1}^{+\infty} \cos \left(2 \pi k c_{\epsilon}\right) e^{-\frac{n^{2} \pi^{2}}{y}}\right)\right) .
\end{aligned}
$$

To compute the $n=0$ mode, the integral of the first term is

$$
\int_{0}^{+\infty} \mathrm{d} y y^{s-\frac{5}{2}} e^{-y v^{2}} \sqrt{\frac{\pi}{y}}=\frac{\sqrt{\pi}}{\left(v^{2}\right)^{s-2}} \Gamma(s-2),
$$

where we used again the definition of the $\Gamma$ function. Now, in order to compute the $n \neq 0$ modes, we can show that 


$$
\begin{aligned}
& \int_{0}^{+\infty} \mathrm{d} y y^{s-\frac{5}{2}} \sqrt{\frac{\pi}{y}} 2 e^{-y v^{2}-\frac{n^{2} \pi^{2}}{y}} \\
& \quad=2 \frac{\sqrt{\pi}}{\left(v^{2}\right)^{s-2}} \int_{0}^{+\infty} \mathrm{d} z z^{s-3} e^{-z-\frac{n^{2} \pi^{2} v^{2}}{y}} .
\end{aligned}
$$

The last integral can be rewritten in terms of the modified Bessel function of the second kind, $K_{v}$ [47]:

$$
\int_{0}^{+\infty} \mathrm{d} t t^{-v-1} e^{-t-\frac{b}{t}}=\frac{2}{b^{v / 2}} K_{v}(2 \sqrt{b}),
$$

so we have

$$
\begin{gathered}
\int_{0}^{+\infty} \mathrm{d} y y^{s-\frac{5}{2}} \sqrt{\frac{\pi}{y}} 2 e^{-y v^{2}-\frac{n^{2} \pi^{2}}{y}} \\
=\frac{2^{2}\left(v^{2}\right)^{2-s} \sqrt{\pi}}{\left(n^{2} \pi^{2} v^{2}\right)^{\frac{2-s}{2}}} K_{2-s}\left(2 n \pi \sqrt{v^{2}}\right) .
\end{gathered}
$$

Defining

$$
I_{n=0}=\lim _{s \rightarrow 0}-\frac{\partial}{\partial s}\left(\frac{\mu^{2 s} T^{4-2 s}\left(v^{2}\right)^{2-s}}{2^{2 s} \pi^{2 s-2} \Gamma(s)} \Gamma(s-2)\right),
$$

and

$$
\begin{aligned}
I_{n \neq 0}= & \lim _{s \rightarrow 0}-\frac{\partial}{\partial s}\left(\frac{\mu^{2 s} T^{4-2 s}\left(v^{2}\right)^{\frac{2-s}{2}}}{2^{2 s-2} \pi^{s} \Gamma(s)}\right. \\
& \left.\times \sum_{n=1}^{+\infty} n^{s-2} K_{2-s}\left(2 n \pi \sqrt{v^{2}}\right) \cos \left(2 \pi n c_{\epsilon}\right)\right),
\end{aligned}
$$

we arrive at

$I=I_{n=0}+I_{n \neq 0}$.

Let us compute $I_{n=0}$ first. Using the properties of the $\Gamma$ function

$\Gamma(s)=(s-1) \Gamma(s-1)=(s-1)(s-2) \Gamma(s-2)$,

then

$I_{n=0}=\lim _{s \rightarrow 0}-\pi^{2} T^{4} v^{4} \frac{\partial}{\partial s}\left(\left(\frac{\Lambda^{-2} T^{2} v^{2}}{2^{-2} \pi^{-2}}\right)^{-s} \frac{1}{(s-1)(s-2)}\right)$.

Taking into account

$\lim _{s \rightarrow 0} \frac{\partial}{\partial s}\left(\frac{\Lambda^{-2} T^{2} v^{2}}{2^{-2} \pi^{-2}}\right)^{-s}=-\ln \left(\frac{\alpha^{2}}{\Lambda^{2}}\right)$,

we have

$I_{n=0}=\frac{\left(\alpha^{2}\right)^{2}}{32 \pi^{2}}\left(\ln \left(\frac{\alpha^{2}}{\Lambda^{2}}\right)-\frac{3}{2}\right)$,

which looks very similar to the standard dimensional regularization procedure used in quantum field theory at zero temperature [48]. Now, in order to compute $I_{n \neq 0}$, we observe that $\Gamma(s)=\frac{1}{s}-\gamma+\mathcal{O}(s)$ when $s \rightarrow 0$, which implies
$\frac{1}{\Gamma(s)}=s+\gamma s^{2}+\mathcal{O}\left(s^{3}\right)$ when $s \rightarrow 0$. So, in the limit $s \rightarrow 0$, there only survives the term which derives from $\frac{1}{\Gamma(s)}$, i.e.,

$I_{n \neq 0}=\frac{\alpha^{2} T^{2}}{\pi^{2}} \sum_{n=1}^{+\infty}(-1)^{n \epsilon+1} n^{-2} K_{2}\left(n \frac{\sqrt{\alpha^{2}}}{T}\right) \cosh (n \mu / T)$.

For the case of fermions,

$$
\begin{aligned}
& I\left(T, \mu, \alpha^{2}\right)=\frac{\left(\alpha^{2}\right)^{2}}{32 \pi^{2}}\left(\ln \left(\frac{\alpha^{2}}{\Lambda^{2}}\right)-\frac{3}{2}\right) \\
& +\frac{\alpha^{2} T^{2}}{\pi^{2}} \sum_{n=1}^{+\infty}(-1)^{n+1} n^{-2} K_{2}\left(n \frac{\sqrt{\alpha^{2}}}{T}\right) \cosh (n \mu / T),
\end{aligned}
$$

while for bosons the result is

$$
\begin{aligned}
I\left(T, \alpha^{2}\right)= & \frac{\left(\alpha^{2}\right)^{2}}{32 \pi^{2}}\left(\ln \left(\frac{\alpha^{2}}{\Lambda^{2}}\right)-\frac{3}{2}\right) \\
& -\frac{\alpha^{2} T^{2}}{\pi^{2}} \sum_{n=1}^{+\infty} n^{-2} K_{2}\left(n \frac{\sqrt{\alpha^{2}}}{T}\right) .
\end{aligned}
$$

\section{Appendix B: Some considerations on the non-monotonic behavior of $P(T)$}

Let us analyze the expression

$$
B=\sum_{i=1}^{4} c_{i} I^{\alpha_{i}}
$$

considered on the quark sector after having determined the cut-off $\Lambda$ requiring $\log Z(0,0)=0 . B$ is simply the sum of the terms containing the Bessel functions. For the sake of clarity we will stop at the first one, $n=1$ :

$$
\begin{aligned}
B= & T^{2} \frac{18}{\pi^{2}}\left[m_{1}^{2} K_{2}\left(\frac{\sqrt{m_{1}^{2}}}{T}\right)+m_{4}^{2} K_{2}\left(\frac{\sqrt{m_{4}^{2}}}{T}\right)\right. \\
& \left.+2 \Re\left(m_{2}^{2} K_{2}\left(\frac{\sqrt{m_{2}^{2}}}{T}\right)\right)\right],
\end{aligned}
$$

where, eventually, $m_{1}^{2}=0.848, m_{2}^{2}=0.2148+0.0579 i, m_{4}^{2}$ $=0.639$. Ignoring the $T^{2}$ term (it is of no importance to the following) and developing around $T=0, B$ is found to be

$$
\begin{aligned}
B \sim & m_{1}^{(3 / 2)} e^{-m_{1} / T}+m_{4}^{(3 / 2)} e^{-m_{4} / T} \\
& +2 \Re\left[\left(m_{2}^{2}\right)^{(3 / 4)} e^{-m_{2} / T}\right] .
\end{aligned}
$$

Thus, with $m_{2}=m_{2 R}+i m_{2 i}$, one gets

$$
\begin{aligned}
B \sim & m_{1}^{(3 / 2)} e^{-m_{1} / T}+m_{4}^{(3 / 2)} e^{-m_{4} / T} \\
& +2 e^{-m_{2 R} / T} \Re\left[\left(m_{2 R}+i m_{2 i}\right)^{(3 / 2)} e^{-i m_{2 i} / T}\right],
\end{aligned}
$$


and defining $m_{2}=m_{2 R}+i m_{2 i}=\rho_{2} e^{i \phi}$ we end up with

$$
\begin{aligned}
B \sim & m_{1}^{(3 / 2)} e^{-m_{1} / T}+m_{4}^{(3 / 2)} e^{-m_{4} / T} \\
& +2 \rho_{2}^{(3 / 2)} e^{-m_{2 R} / T} \cos \left(\frac{m_{2 i}}{T}+\frac{3}{2} \phi\right) .
\end{aligned}
$$

Now, it is clear that, when $T \rightarrow 0, B$ oscillates due to the presence of the cos function, that is to say, because of the complex masses. Thus, if $m_{2 i}$ is zero, $B$ is strictly monotonic. This is exactly what one can get, for example, changing by $+9 \%$ the parameters. Indeed, in this case, using $M_{3}=0.214 \mathrm{GeV}^{3}, m^{2}=0.697 \mathrm{GeV}^{2}, m_{0}=0.015 \mathrm{GeV}$, we get $\alpha_{1}=0.194, \alpha_{2}=0.283, \alpha_{3}=0.916 \mathrm{GeV}^{2}$; see Eq. (3). While, for the gluons, taking $N^{2}=2.74 \mathrm{GeV}^{2}, m^{2}=$ $-2.09 \mathrm{GeV}^{2}, \lambda^{4}=5.8 \mathrm{GeV}^{4}$ in Eq. (18), we get $r_{+}=$ $0.55, r_{-}=0.09 \mathrm{GeV}^{2}$. Obviously, as the presence of real poles over complex conjugated poles only depends on the discriminant of (18), one can just vary one of the three parameters keeping fixed the other two in such a way as to change the sign of the discriminant itself (in the case of the quark propagator the analysis of the roots is more complicated as it involves a cubic equation, but, conceptually, a similar scheme can be applied). However, this is quite beyond the scope of the present work as our intention was just to emphasize that with real poles (despite the violation of positivity related to the negative residue) one can solve the above mentioned pathological behavior of the equation of state $P=P(e)$. The reason is that the main goal of the present paper is the analysis of the lattice propagators both of which (quarks and gluons) strongly favor complex conjugated poles.

Is it possible to obtain a strictly monotonic function even in the presence of two complex conjugate masses? No, in this case it is possible to ensure that $B$ is positive. For instance, reasonable conditions to achieve this are either $m_{1}<m_{2 R}$, or $m_{4}<m_{2 R}$. Indeed, in these cases, assuming for example $m_{4}<m_{2 R}$ and $2\left(\frac{\rho_{2}}{m_{4}}\right)^{(3 / 2)}<1$, one gets

$$
\begin{aligned}
B \sim & m_{4}^{(3 / 2)} e^{-m_{4} / T}\left[1+\left(\frac{m_{1}}{m_{4}}\right)^{(3 / 2)} e^{\frac{-m_{1}+m_{4}}{T}}\right. \\
& \left.+2\left(\frac{\rho_{2}}{m_{4}}\right)^{(3 / 2)} e^{\frac{-m_{2 R}+m_{4}}{T}} \cos \left(\frac{m_{2 i}}{T}+\frac{3}{2} \phi\right)\right] \geq 0 .
\end{aligned}
$$

However, there is no possibility to obtain a strictly monotonic function. The same will be true for the gluonic sector. In this appendix we presented a concrete example of how the propagator changes when one moves to real poles but, of course, our main goal is to disclose the thermodynamical pathologies related to the complex poles supported by the lattice data and the necessity to include in the thermodynamical description extra degrees of freedom in order to avoid such pathologies

We conclude that in the presence of complex conjugated poles, the thermodynamical quantities as pressure and entropy are not well behaved in some region, as was already pointed out in [49]. Thus, the above considerations strongly suggest that some extra physical parameter is necessary to properly label the equilibrium states. There are many interesting options such as flavor parameters (in the case of quarks propagator), group parameters, and so on.

\section{References}

1. J. Greensite, An Introduction to the Confinement Problem. Lecture Notes in Physics (Springer, Berlin, 2011)

2. V.N. Gribov, Nucl. Phys. B 139, 1 (1978)

3. L.S. Brown, G.J. MacLay, Phys. Rev. D 184, 1272 (1969)

4. J.S. Dowker, R. Critchley, Phys. Rev. D 13, 3224 (1976)

5. S.W. Hawking, Commun. Math. Phys. 55, 133 (1977)

6. E. Ostgaard, Phys. Rep. 242, 313-332 (1994)

7. D. Blaschke, D. Sedrakian, Superdense QCD Matter and Compact Stars. NATO Science Series (Springer, Berlin, 2003)

8. C. Rhoades, R. Ruffini, Phys. Rev. Lett. 32, 324 (1972)

9. M.S. Guimaraes, B.W. Mintz, L.F. Palhares, Phys. Rev. D 92, 085029 (2015)

10. A. Actor, Phys. Lett. B 157, 153 (1985)

11. A. Actor, Nucl. Phys. B 265, 689 (1986)

12. M.B. Parappilly, P.O. Bowman, U.M. Heller, D.B. Leinweber, A.G. Williams, J.B. Zhang, Phys. Rev. D 73, 054504 (2006)

13. M.S. Bhagwat, M.A. Pichowsky, C.D. Roberts, P.C. Tandy, Phys. Rev. C 68, 015203 (2003)

14. L. Baulieu, M.A.L. Capri, A.J. Gomez, V.E.R. Lemes, R.F. Sobreiro, S.P. Sorella, Eur. Phys. J. C 66, 451 (2010)

15. D. Dudal, M.S. Guimaraes, L.F. Palhares, S.P. Sorella, Ann. Phys. 365, 155-179 (2016). arXiv:1303.7134 [hep-ph]

16. J.I. Kapusta, C. Gale, Finite-Temperature Field Theory Principles and Applications (Cambridge University Press, New York, 2006)

17. A. Cucchieri, T. Mendes, PoS LAT2007 (2007) 297

18. R.F. Sobreiro, S.P. Sorella, Introduction to the Gribov Ambiguities In Euclidean Yang-Mills Theories. arXiv:hep-th/0504095 (unpublished)

19. F. Canfora, A. Giacomini, J. Oliva, Phys. Rev. D 82, 045014 (2010)

20. A. Anabalon, F. Canfora, A. Giacomini, J. Oliva, Phys. Rev. D 83, 064023 (2011)

21. F. Canfora, A. Giacomini, J. Oliva, Phys. Rev. D 84, 105019 (2011)

22. F. Canfora, P. Salgado-Rebolledo, Phys. Rev. D 87(4), 045023 (2013)

23. M. de Cesare, G. Esposito, H. Ghorbani, Phys. Rev. D 88, 087701 (2013)

24. F. Canfora, F. de Micheli, P. Salgado-Rebolledo, J. Zanelli, Phys. Rev. D 90, 044065 (2014)

25. F. Canfora, M. Kurkov, L. Rosa, P. Vitale, JHEP 1601, 014 (2016)

26. D. Zwanziger, Nucl. Phys. B 321, 591 (1989)

27. D. Zwanziger, Nucl. Phys. B 323, 513 (1989)

28. D. Zwanziger, Nucl. Phys. B 399, 477 (1993)

29. G.F. Dell'Antonio, D. Zwanziger, Nucl. Phys. B 326, 333 (1989)

30. M. Maggiore, M. Schaden, Phys. Rev. D 50, 6616 (1994)

31. J.A. Gracey, JHEP 0605, 052 (2006)

32. D. Dudal, R.F. Sobreiro, S.P. Sorella, H. Verschelde, Phys. Rev. D 72, 014016 (2005)

33. D. Dudal, S.P. Sorella, N. Vandersickel, H. Verschelde, Phys. Rev. D 77, 071501 (2008)

34. D. Dudal, S.P. Sorella, N. Vandersickel, Eur. Phys. J. C 68, 283 (2010)

35. D. Dudal, M.S. Guimaraes, S.P. Sorella, Phys. Rev. Lett. 106, 062003 (2011)

36. F. Canfora, L. Rosa, Phys. Rev. D 88, 045025 (2013)

37. D. Zwanziger, Phys. Rev. D 76, 12504 (2007) 
38. F. Canfora, P. Pais, P. Salgado-Rebolledo, Eur. Phys. J. C 74, 2855 (2014)

39. P. Cooper, D. Zwanziger, Phys. Rev. D 93, 105026. arXiv:1512.08858 [hep-th]

40. F. Canfora, D. Dudal, I. Justo, P. Pais, L. Rosa, V. Vercauteren, Eur. Phys. J. C. 75, 326 (2015)

41. A. Cucchieri, D. Dudal, T. Mendes, N. Vandersickel, Phys. Rev. D 85, $094513(2012)$

42. D. Dudal, S.P. Sorella, N. Vandersickel, H. Verschelde, Phys. Lett. B 680, 377 (2009)

43. D. Dudal, J.A. Gracey, S.P. Sorella, N. Vandersickel, H. Verschelde, Phys. Rev. D 78, 125012 (2008)

44. M.A.L. Capri, D. Dudal, A.J. Gomez, M.S. Guimaraes, I.F. Justo, S.P. Sorella, D. Vercauteren, Phys. Rev. D 88, 085022 (2013)
45. M.A.L. Capri, D. Dudal, A.J. Gomez, M.S. Guimaraes, I.F. Justo, S.P. Sorella, Eur. Phys. J. C 73, 2346 (2013)

46. U. Reinosa, J. Serreau, M. Tissier, N. Wschebor, Phys. Rev. D 91, 045035 (2015)

47. L. Glasser, K.T. Kohl, C. Koutschan, V.H. Moll, A. Straub, The integrals in Gradshteyn and Ryzhik. Part 22: Bessel- $K$ functions. Sci. Ser. A: Math. Sci. 22, 129-151 (2012)

48. M.E. Peskin, D.V. Schroeder, An Introduction to Quantum Field Theory (Perseus Books Publishing L.L.C, New York, 1995)

49. S. Benić, D. Blaschke, M. Buballa, Phys. Rev. D 86, 074002 (2012). arXiv:1206.6582 [hep-ph] 